\title{
Numerical simulation of wave interacting with a free rolling body
}

\author{
Jae Hwan Jung ${ }^{1}$, Hyun Sik Yoon ${ }^{2}$, Ho Hwan Chun ${ }^{2}$, Inwon Lee ${ }^{2}$ and Hyun Park ${ }^{2}$ \\ ${ }^{I}$ Department of Naval Architecture and Ocean Engineering, Pusan National University, Busan, Korea \\ ${ }^{2}$ Global Core Research Center for Ships and Offshore Plants, Pusan National University, Busan, Korea
}

\begin{abstract}
The present study numerically models the interaction between a regular wave and the roll motion of a rectangular floating structure. In order to simulate two-dimensional incompressible viscous two-phase flow in a numerical wave tank with the rectangular floating structure, the present study used the volume of fluid method based on the finite volume method. The sliding mesh technique is adopted to handle the motion of the rectangular floating structure induced by fluid-structure interaction. The effect of the wave period on the flow, roll motion and forces acting on the structure is examined by considering three different wave periods. The time variations of the wave height and the roll motion of the rectangular structure are in good agreement with experimental results for all wave periods. The present response amplitude operator is in good agreement with experimental results with the linear potential theory. The present numerical results effectively represent the entire process of vortex generation and evolution described by the experimental results. The longer wave period showed a different mechanism of the vortex evolution near each bottom corner of the structure compared to cases of shorter wave periods. In addition, the $x$-directional and z-directional forces acting on the structure are analyzed.
\end{abstract}

KEY WORDS: Wave; Roll motion; Numerical wave tank; Viscous two-phase flow.

\section{INTRODUCTION}

In the past several years offshore structures have been moved to deeper water, due to increasing interest in the development of subsea resources. Offshore structures can undergo large-amplitude motion due to harsh environments and extremely steep waves. Responses of floating bodies such as ships or offshore structures to incident waves are one of the main concerns in ocean engineering.

Offshore structures have been investigated in terms of six degree of freedom motion to evaluate design that can meet safety standards. As a result, there is an increasing interest in the use of numerical simulation to study the interaction between waves and structures. One of the most critical aspects of motions of a structure is roll, which is of practical interest for both safety and comfort reasons.

Roll motion, unlike other motions, is highly nonlinear because of the roll damping effect. However, the ability to predict roll motion lags considerably behind that of motions such as heave and pitch. Potential flow theories, based on the assumptions that the flow is inviscid and irrotational, can introduce large errors. They fail to account for viscous damping, flow separation, and vortex generation (Wehausen, 1971).

This shortfall is normally compensated by introducing a viscous roll damping coefficient. Traditionally, hydrodynamic coefficients used in the prediction of rolling motions have been obtained from empirical formulas based on experimental test results (Himeno, 1981; Ikeda and Himeno, 1981). However, it is not easy to find a proper viscous damping coefficient, or to generalize data for roll damping.

Corresponding author: Hyun Sik Yoon

e-mail:lesmodel@pusan.ac.kr 
Despite some difficulties, numerous numerical methods have been developed to simulate viscous flow. Faltinsen and Pettersen (1987) studied the separated flow and vortex generation of a blunt body and rectangularly shaped body using the vortex tracking method. Scolan and Faltinsen (1994) investigated separated flow from bodies with sharp corners using the vortex in cell method. Yeung and Vaidhyanathan (1994) considered separated flows near a free surface using the random vortex method. Unfortunately, these numerical methods require a priori knowledge of the boundary layer separation point, and are therefore difficult to apply for various problems.

Recently, many researchers have used techniques based on the solution of Reynolds averaged Navier-Stokes (RANS) equations (Korpus and Falzarano, 1997; Sarkar and Vassalos, 2000; Chen et al., 2002; Wilson et al., 2006). These methods naturally incorporate the effects of viscosity with a turbulence model, and can easily be extended to various practical problems.

Most of the above publications are concerned with problems, either with fixed bodies or forced motion. Studies of the interaction between waves and free-rolling bodies are still very limited in the available literature.

Jung et al. (2004a) experimentally investigated wave interactions with a fixed rectangular structure using particle image velocimetry (PIV) to simulate the condition of a barge in a beam sea. The mean velocity field was demonstrated along with the generation and evolution of vortices on both sides of the structure. In subsequent studies, Jung (2004b) and Jung et al. (2005) released the roll motion of the structure to investigate the two-dimensional flow characteristics of wave interactions with freerolling rectangular structures using PIV.

In this study, we simulated the coupled interaction between a wave and a rectangular structure in rolling motion. The main purpose of the present study is the verification of a numerical method that describes wave and body interaction. The time variations of wave height and the roll motion of the rectangular structure were validated by comparison with experimental results of Jung et al. (2004a). The characteristics of flow, roll motion and the forces acting on the structure were analyzed for different wave periods.

\section{NUMERICAL DETAILS}

\section{Numerical approach}

The present two-dimensional problem of wave-structure interaction is governed by the Navier-Stokes equations and continuity equation. Once the Reynolds averaging approach for turbulence modeling is applied, the Navier-Stokes equations can be written in Cartesian tensor form as

$$
\begin{gathered}
\frac{\partial}{\partial x_{i}}\left(\rho u_{i}\right)=0 \\
\frac{\partial}{\partial t}\left(\rho u_{i}\right)+\frac{\partial}{\partial x_{j}}\left(\rho u_{i} u_{j}\right)= \\
-\frac{\partial p}{\partial x_{i}}+\frac{\partial}{\partial x_{j}}\left[\mu\left(\frac{\partial u_{i}}{\partial x_{j}}+\frac{\partial u_{j}}{\partial x_{i}}\right)\right]+\frac{\partial}{\partial x_{j}}\left(-\rho \overline{u_{i}^{\prime} u_{j}^{\prime}}\right)+F_{i}
\end{gathered}
$$

where $x_{i}$ are Cartesian coordinates, $u_{i}$ are the corresponding velocity components, $p$ is the pressure, $\rho$ is the density, $\mu$ is the viscosity, and $F_{i}$ is an external body force (e.g. gravity). Also, $-\rho \overline{u_{i}^{\prime} u_{j}^{\prime}}$ is the Reynolds stress term that was closed by using the standard $k-\varepsilon$ turbulence model (Launder and Spalding, 1972).

The equations of motion of a rectangular structure are expressed as follows,

$$
\begin{aligned}
& \vec{F}=m \frac{d \vec{V}}{d t} \\
& \vec{L}=\frac{d}{d t}\left(I_{0} \vec{\omega}\right)
\end{aligned}
$$


where $\vec{F}$ and $\vec{L}$ are the hydrodynamic force and the moment of the hydrodynamic force acting on the structure, respectively. $\vec{V}$ and $\vec{\omega}$ are the velocity and angular velocity of the floating structure, respectively. $\mathrm{m}$ is the mass of the structure, $I_{0}$ is the moment of inertia of the structure at the center of gravity in a space fixed coordinate system.

In this study, the volume of fluid (VOF) method was employed to capture the free surface. The VOF method is popularly adopted to track and capture the free surface in two-phase problem. Also, most commercial computational fluid dynamics (CFD) codes use a variation of the VOF approach. In each cell, the volume fraction $\left(Q_{\delta}\right)$ of $\delta$ th fluid is introduced because the VOF method is designed for two or more immiscible fluids. In each cell, the sum of the volume fractions of all phases is unity ( $\sum_{\delta=1}^{n} Q_{\delta}=1$ ). A cell with a $Q_{\delta}$ value (scalar quantity) of 0 is void, and a value of 1 represents a full cell. The cell contains a free surface if the $Q_{\delta}$ value is between 0 and 1 . At each time step, a transport equation is solved to find the distribution of the fluid. At each time step, the distribution of the fluid is found by solving the following transport equation:

$$
\frac{\partial Q_{\delta}}{\partial t}+u_{i} \frac{\partial Q_{\delta}}{\partial x_{i}}=0
$$

A single momentum equation is solved throughout the domain, and the resulting velocity field is shared among the phases (Hirt and Nichols, 1981). Convection and diffusion terms are discretized using the quadratic upstream interpolation for convective kinematics (QUICK) scheme and the second-order accurate central differencing scheme, respectively. For unsteady flow calculations, time derivative terms are discretized using the first-order accurate backward implicit scheme. The velocitypressure coupling and overall solution procedure are based on a pressure implicit with splitting of operators (PISO) algorithm adapted to the structured grid. The commercial CFD package, FLUENT (2010) was used for all numerical predictions in present study. Further details of the implementation can be found in the FLUENT (2010) manuals.

\section{Computational domain and grid system}

A rectangular structure $0.3 m$ wide $(\mathrm{B})$, and $0.1 \mathrm{~m}$ high $(\mathrm{H})$ was used in the numerical simulation. The structure was fixed at the center of gravity (CG). The structure was located $10 \mathrm{~m}$ from the wave maker, although the experimental condition used by Jung (2004b) is $20 \mathrm{~m}$. The initial water height was $0.9 \mathrm{~m}$ and a sloping beach was installed at the end of the wave tank to absorb the wave energy and reduce reflection as shown Fig. 1.

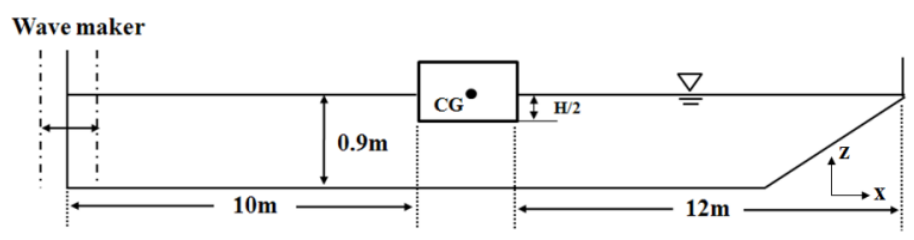

Fig. 1 Initial geometry of numerical wave tank and rectangular barge.

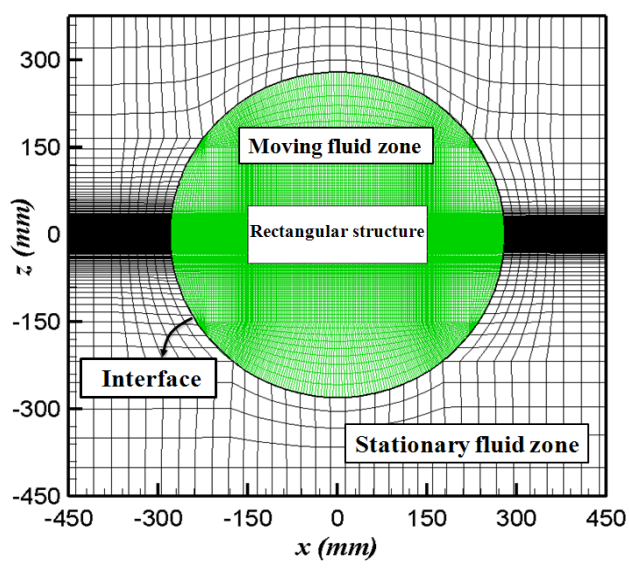

Fig. 2 Grid system around rectangular structure. 
A piston-type wave generator was incorporated in the computational domain to generate the desired incident waves. The layering mesh technique, which is suitable for translation motion was used to move the piston. The piston motion was pure surge (translation in the x-direction only) and followed a the sinusoidal function given by

$$
S(t)=A \cdot \sin (\omega t)
$$

where $A$ and $\omega$ are the wave amplitude and frequency, respectively. The wave amplitude was changed from different wave conditions in this study.

To simulate wave interaction with the structure, the sliding mesh technique was used to characterize the motion of the rectangular structure induced by fluid-structure interaction. Two independent grid systems were generated based on the structured grid as shown in Fig. 2. The moving fluid zone, including the rectangular structure, was allowed to rotate due to the moment caused by the hydrodynamic force acting on the structure. The stationary fluid zone was fixed. These two flow fields were interpolated each other through the interface by using a linear interpolation method. The grid was refined near the structure and the free surface to achieve better resolution of the flow field. Three different grid systems (coarse, medium and fine grids having $60,000,100,000$ and 150,000 grid cells, respectively) were considered to test the grid dependence of the solutions and to validate the present numerical methods. The dependence of the solutions on the grid systems considered in this study were not strong. Consequently, the medium grid system was selected for all cases.

\section{Wave conditions}

The present study considered three different wave periods that were shorter, equal to and longer than the roll natural period of the rectangular structure in order to observe the dependence of the flow structures around the rectangular floating structure and its roll motion on the wave period. The roll natural period of the structure was obtained by conducting a roll free decay test. Table 1 shows that the wave conditions used in present computation are the same as those used by Jung (2004b).

Table 1 Wave conditions.

\begin{tabular}{|c|c|c|c|}
\hline & Wave period, $T_{\mathrm{s}}(s)$ & Wave height, $\mathrm{h}(\mathrm{m})$ & Wave length, $\lambda(\mathrm{m})$ \\
\hline Case 1 $\left(T_{s}=T_{n}\right)$ & 0.93 & 0.027 & 1.35 \\
\hline Case 2 $\left(T_{s}<T_{n}\right)$ & 0.8 & 0.029 & 1.0 \\
\hline Case 3 $\left(T_{s}>T_{n}\right)$ & 1.2 & 0.06 & 2.2 \\
\hline
\end{tabular}

\section{RESULTS AND DISCUSSION}

\section{Wave and roll motion profiles}

Fig. 3 shows a comparison of the profiles of wave elevation $(\eta)$ and the roll angle of the rectangular structure $(\phi)$ according to phases of velocity field with experimental results by Jung (2004b) for different wave periods of $T_{s}=0.93 \mathrm{~s}, T_{s}=0.8 \mathrm{~s}$ and $T_{s}=1.2 \mathrm{~s}$. Regardless of the wave periods, the present results are in good agreement with those of the experiment with respect to the pattern, the value of the wave height, and the roll motion of the rectangular structure. The largest amplitude of $\phi$ appeared at $T_{s}=0.93 \mathrm{~s}$ due to the resonance effect, since this wave period was very close to the roll natural period of the rectangular structure.

In the design of ship and other floating structures, a response amplitude operator (RAO) is an engineering statistic, or a set of such statistics, used to determine the likely behavior of a ship when operating at sea. RAOs are usually obtained using models of proposed ship designs tested in a model basin, or by running CFD programs, or from linear potential theory. 


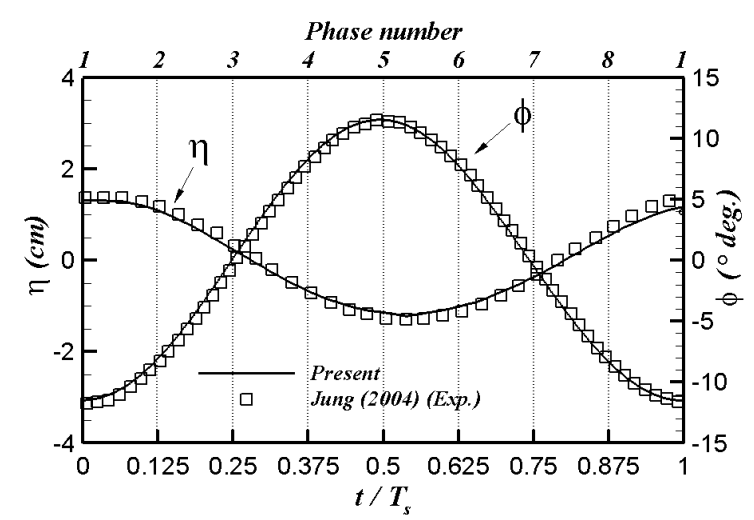

(a)

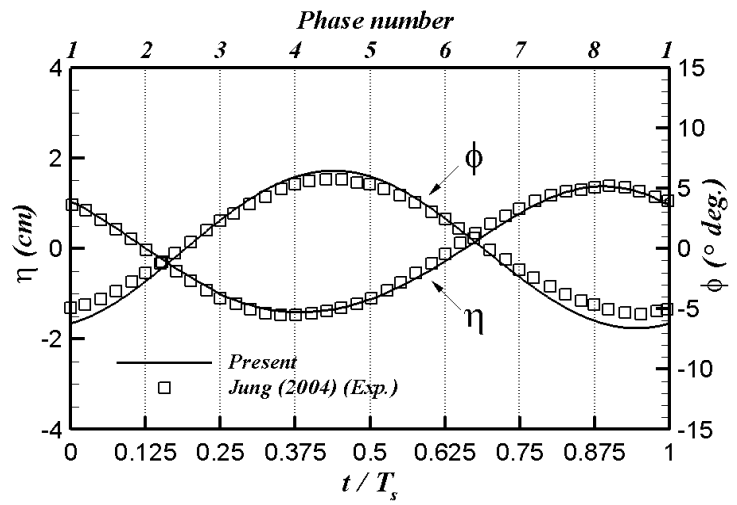

(b)

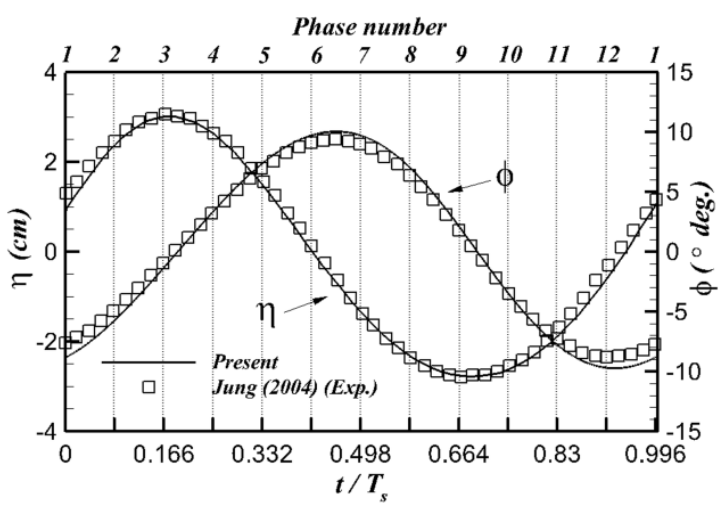

(c)

Fig. 3 Time histories of wave elevation $(\eta)$ and rotating angle of the rectangular structure $(\phi)$ with phases of velocity field at different wave periods of (a) $T_{s}=0.93 \mathrm{~s}$, (b) $T_{s}=0.8 \mathrm{~s}$ and (c) $T_{s}=1.2 \mathrm{~s}$.

Fig. 4 shows a comparison of present RAOs with the experimental results and linear potential theory results by Jung (2004b) as a function of $\omega / \omega_{N}$, where $\omega$ and $\omega_{N}$ are the exciting frequency and the roll natural frequency of the structure, respectively. The RAO is defined as $\phi / k \zeta_{A}$ where $\phi$ and $k \zeta_{A}$ are a roll angle and the wave slope, respectively. The RAO values for different wave frequencies are in excellent agreement with those from Jung's experiment (2004b). Also, RAO values are in good agreements with linear potential theory for the higher frequency waves, but underestimate for the lower frequency waves (Salvesen et al., 1970; Downie et al., 1988). However, the RAO based on theory is significantly magnified at the natural frequency, since linear potential theory includes only wave making damping but not viscous damping.

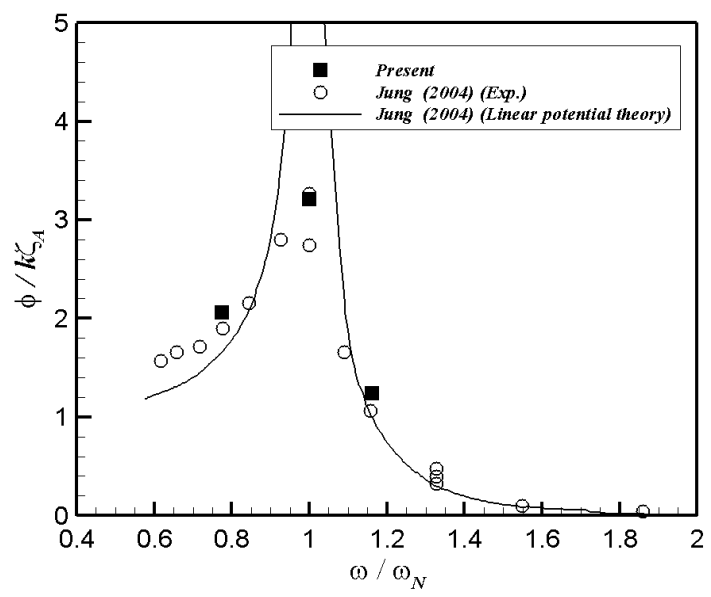

Fig. 4 Response amplitude operator (RAO) for roll motion. 


\section{Flow structures}

Fig. 5 shows the velocity vectors and vorticity contours of present numerical results and experimental results of Jung (2004b) according to phase, as shown in Fig. 3(a), for $T_{s}=0.93 \mathrm{~s}$. In phase 1, when the rotational angle of the structure reached almost its maximum value in the negative rotational direction, positive vortices rotating counterclockwise were formed near the left bottom and the right side wall owing to flow separation at the left and right bottom corners, respectively. Thus, the maximum positive vorticities appeared in these regions close to the bottom corners, as shown in Fig. 5(a). The velocity vectors and vorticity contours of the present computational results are reasonably close to the experimental results (Jung, 2004b).

In phase 2 , the structure started to rotate counterclockwise. The magnitude of the rotational angle decreased with the negative value of $\phi$ as shown in Fig. 3(a). In this phase, the positive vortex observed in phase 1 near the bottom corner of the seaward side disappeared because the counter-rotating motion of the structure to the positive vortex restricted the vortex motion. However, weak positive vorticity still remained. In contrast to the seaward bottom corner, the clockwise rotating vortex developed early near the leeward bottom corner, as shown in Fig. 5(b). The consistent results from the present computations and the previous experiment (Jung, 2004b) confirm that the present results are in good qualitative and quantitative agreement with the experimental results.
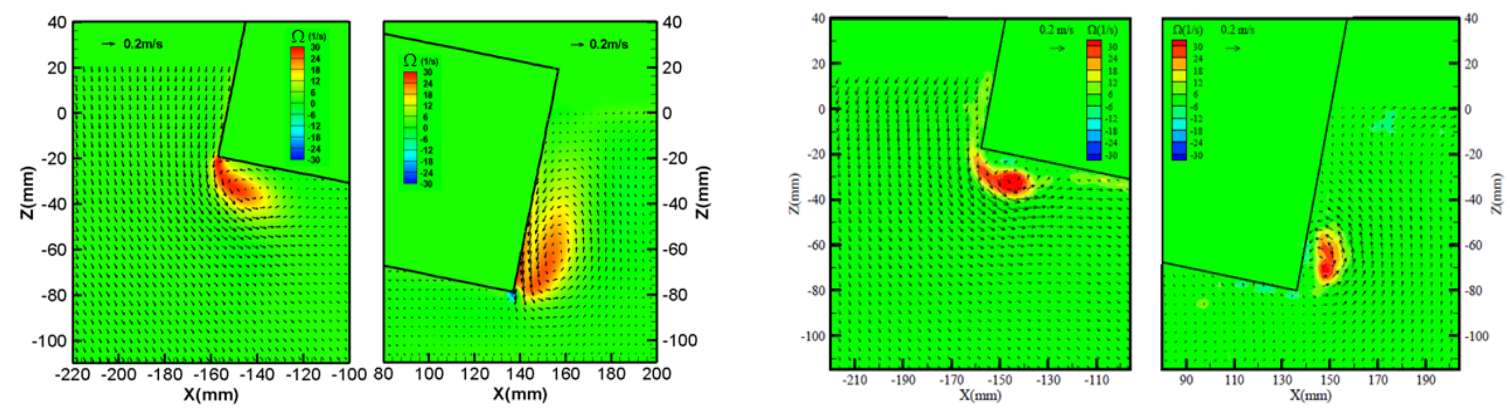

(a)
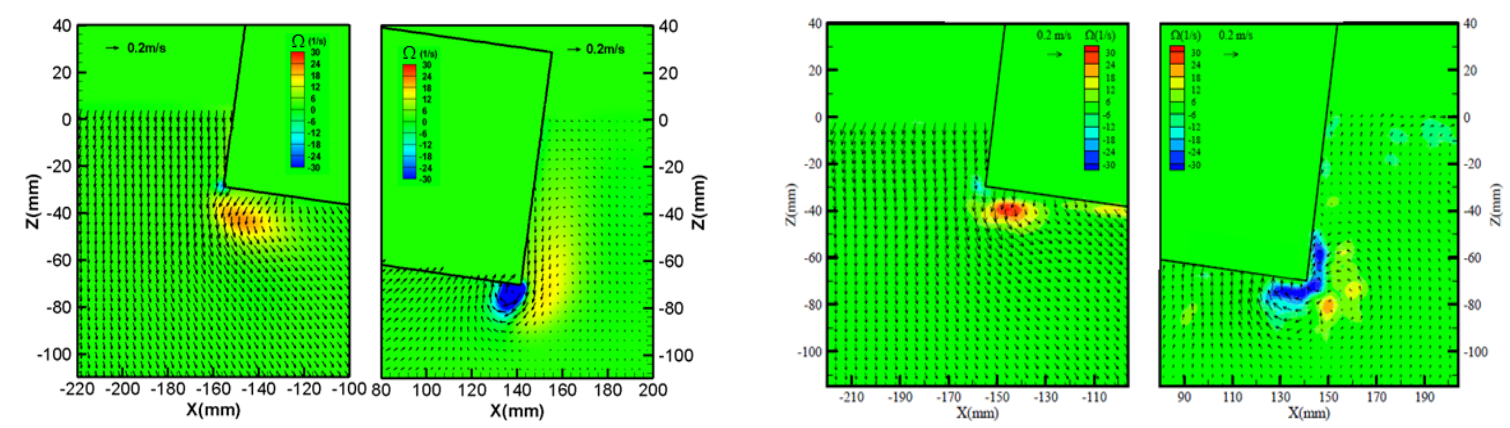

(b)
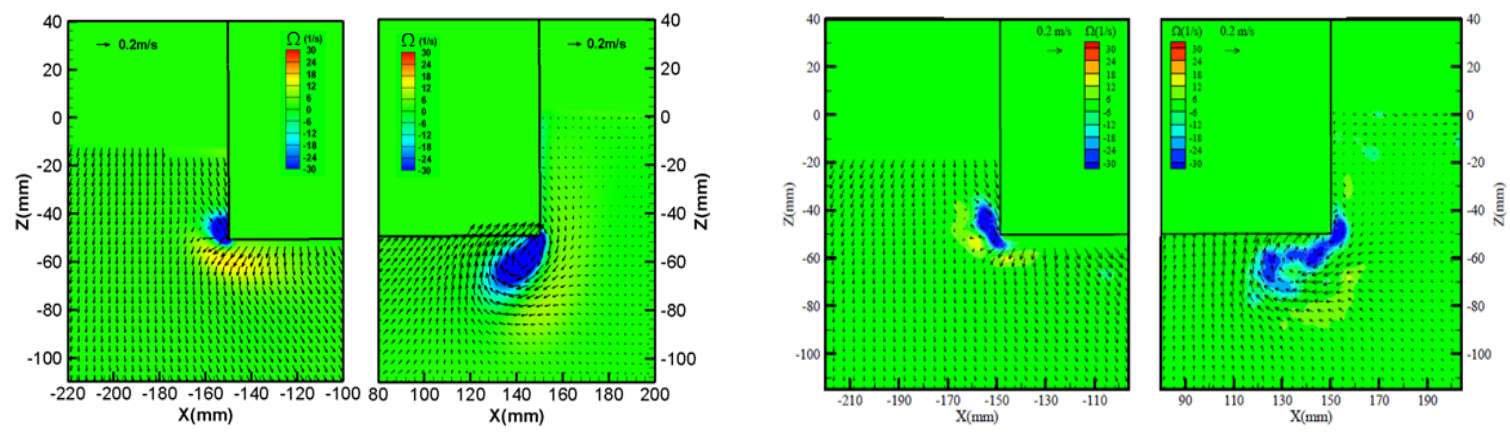

(c)

Fig. 5 Velocity field of $T_{s}=0.93 \mathrm{~s}$ wave : phase number of each subtitle matches to the phase in Fig. 3(a). (left column : Present (CFD), right column : Jung (2004) (Exp.)). 

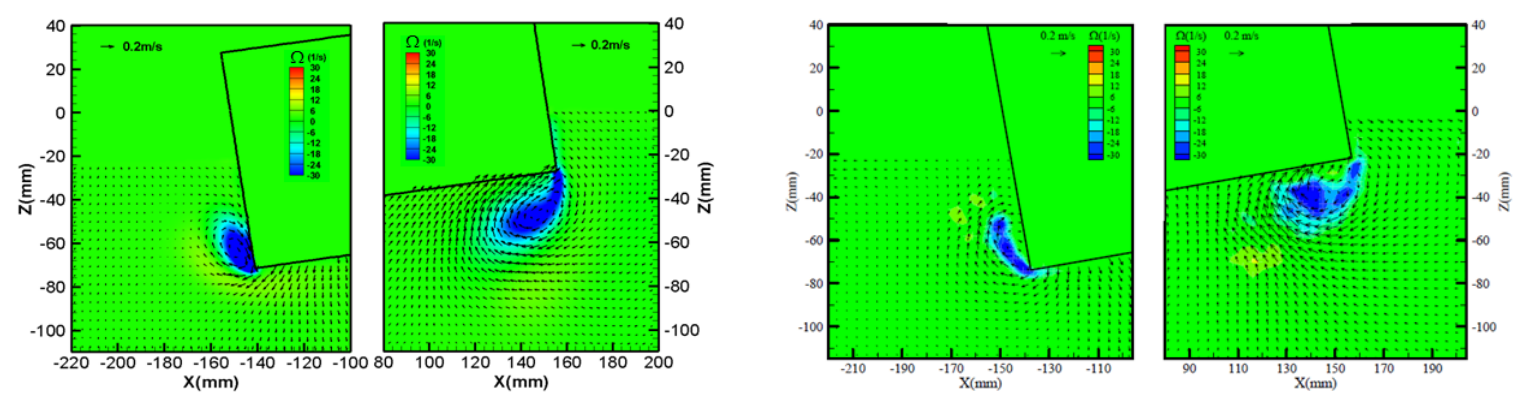

(d)
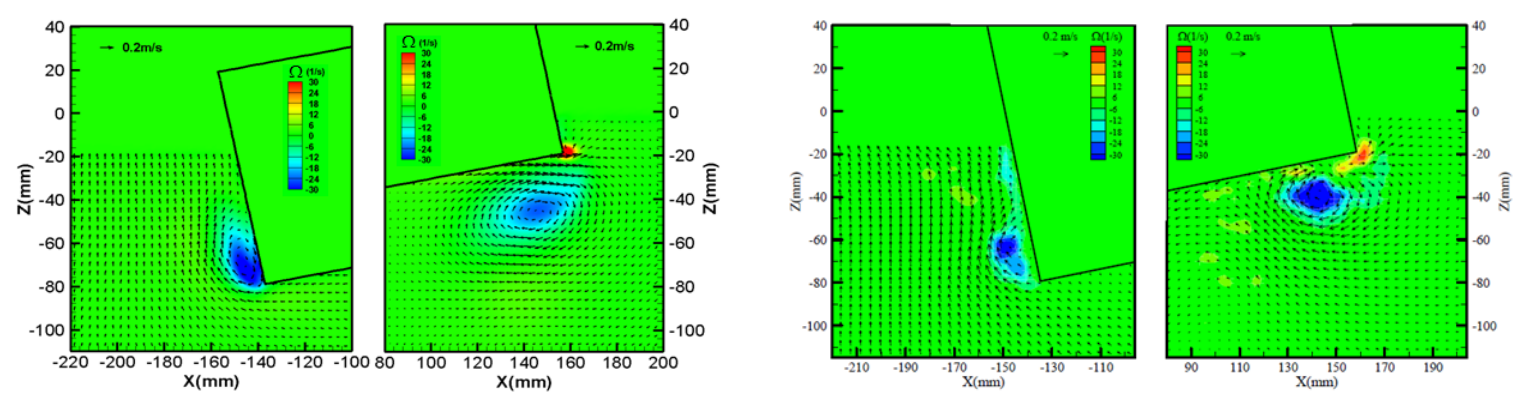

(e)
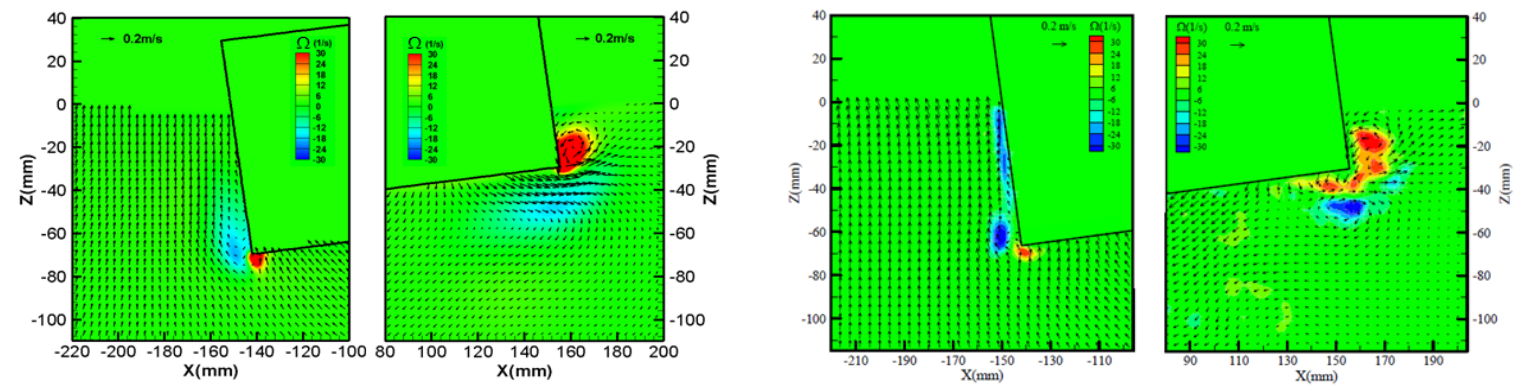

(f)
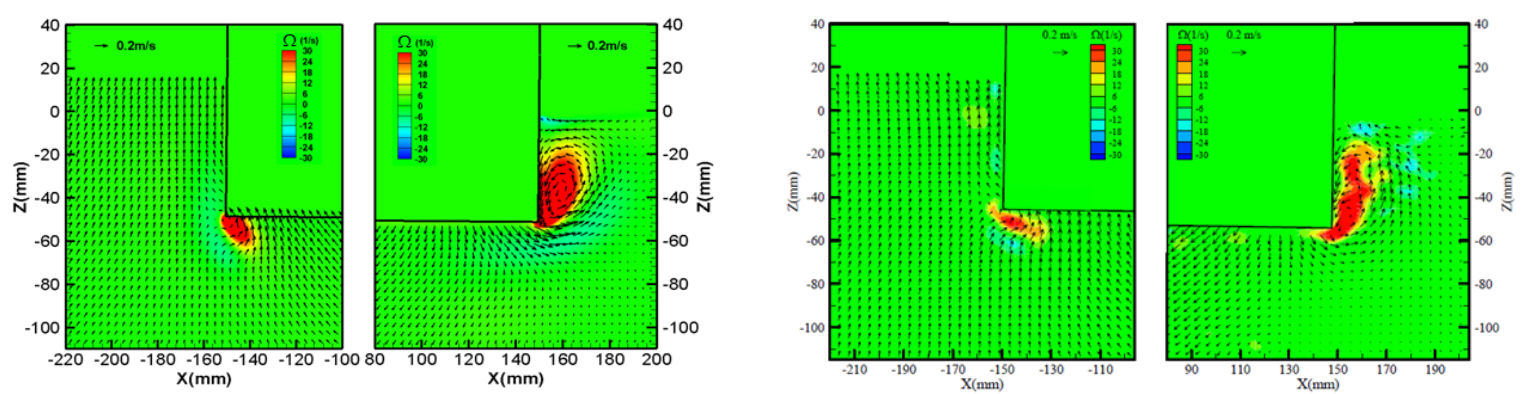

(g)
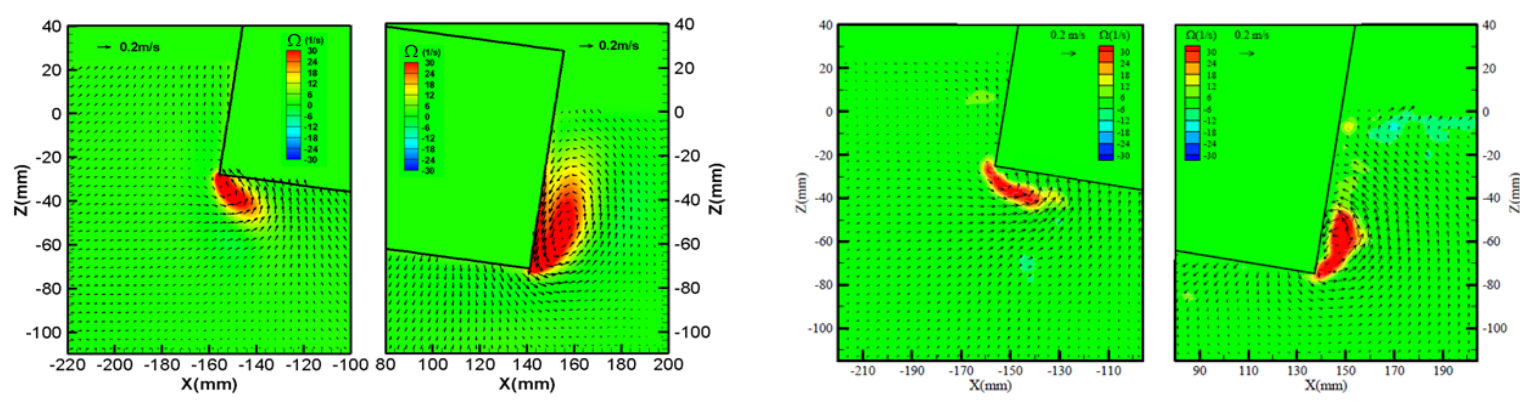

(h)

Fig. 5 Continued. 
In phase 3, the structure is continuously rotating in the counterclockwise direction and is placed almost horizontally with a rotational angle of about $0^{\circ}$, as shown in Fig. 3(a). The clockwise rotating vortex appeared near the left side wall at the seaward bottom corner. On the leeward side, the clockwise rotating vortex that originated early in phase 2 increased in size and strength, as shown in Fig. 5(c). In phase 4, these clockwise rotating vortices on both sides kept augmenting with an increasing counterclockwise roll of the structure, Figs. 5(c) and (d). In phase 4, the positive vorticities near both sides also dissipated.

In phase 5, since the structure reached the extreme positive rotational angle as shown in Fig. 3(a), the clockwise rotating vortex near the right bottom corner on the leeward side of the structure moved further away from the structure. Subsequently, the negative vorticity became weak and separated from the left bottom corner, as shown in Fig. 5(e). This can be verified by comparing phase 4 in Fig. 5(d) and phase 5 in Fig. 5(e). Simultaneously, in the vicinity of the right corner on the leeward side, a tiny positive vorticity started to appear. On the seaward side, the negative vorticity accompanied by the clockwise rotating vortex near the left side corner also became weak in comparison to phase 4 . In contrast to behavior on the leeward side, the positive vorticity did not exit at this phase on the seaward side.

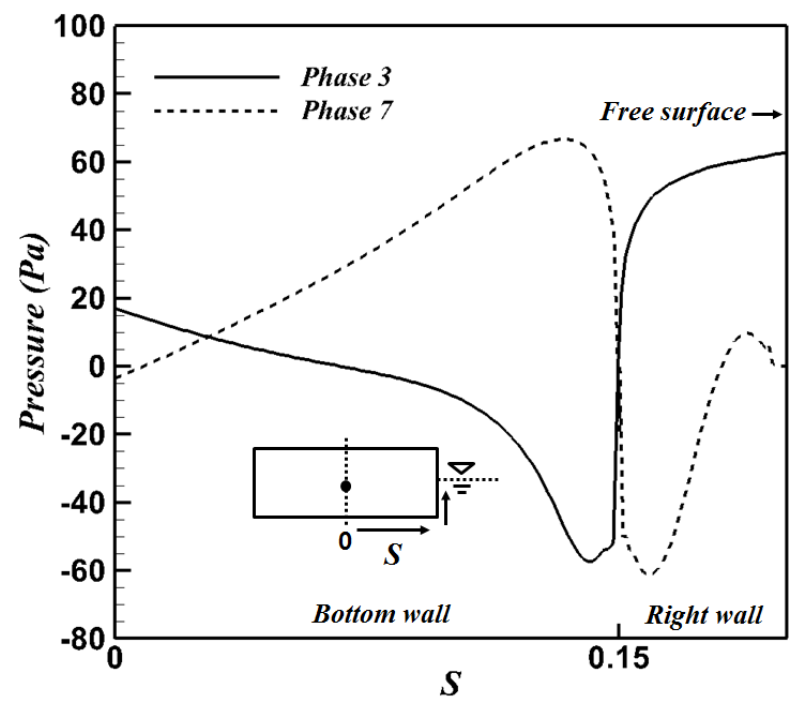

Fig. 6 Pressure distributions along the structure surface in phases 3 and 7 at $T_{s}=0.93 \mathrm{~s}$.

The structure kept rotating clockwise, corresponding to phases 6, 7 and 8 shown in Figs. 5(f), (g) and (h), respectively. The positive vortex increased with increasing magnitude of the positive vorticity in the corner of the leeward side. The negative vorticity decreased and eventually disappeared near the bottom corner of the leeward side in phase 7, as shown in Fig. 5(g). A small positive vortex started to generate in the corner of the seaward side, starting its clockwise roll motion in phase 6 as shown in Fig. 5(f). Then, the positive vortex came into the larger vortex shape on the seaward side until the start of phase 8 , as shown in Fig. 5(h).

This entire process, described above, of the vortex generation and evolution was made by roll motion ahead of the fluid near the structure. Therefore, it caused the viscous damping effect, which reduces the roll motion with the roll natural period wave. This viscous damping effect related to the corner vortices is associated with the pressure force on the body and eventually the roll motion of the body. Thus, the pressure distribution on the body surface is plotted to identify the viscous damping effect on the force in Fig. 6 where considers representatively phase 3 and phase 7 corresponding to Figs. 5(c) and (g), respectively. In addition, the hydrostatic pressure is ignored to observe the net effect of the vortices and the right half of the body is only considered because of the almost symmetric relation between the left and right half.

In phase 3, the negative peak of the pressure near the right bottom corner distinctly appears as shown in Fig. 6, which is derived by the strong clockwise rotating vortex at this corner as observed in Fig. 5(c). In this phase, this negative pressure plays a role in damping the counterclockwise roll motion of the body. 
In phase 7 in Fig. 6, the negative peak of the pressure occurs at the position near the right corner on the right side wall, which is associated with the strong counterclockwise rotating vortex at this region as shown in Fig. 5(g). This suction effect interrupts the clockwise roll motion of the body.

Fig. 7 shows the velocity vectors and vorticity contours at different phases for a wave period of $T_{s}=0.8 \mathrm{~s}$, which is shorter than the roll natural period. In general, the flow pattern according to the rotational angle was about the same as that of $T_{s}=$ $0.93 s$ shown in Fig. 5 .

When the structure started to incline in the counterclockwise direction in phase 1 (Fig. 3(b)), a counterclockwise vortex with positive vorticity appeared to be most developed under the bottom of structure on the seaward side, as shown in Fig. 7(a). While

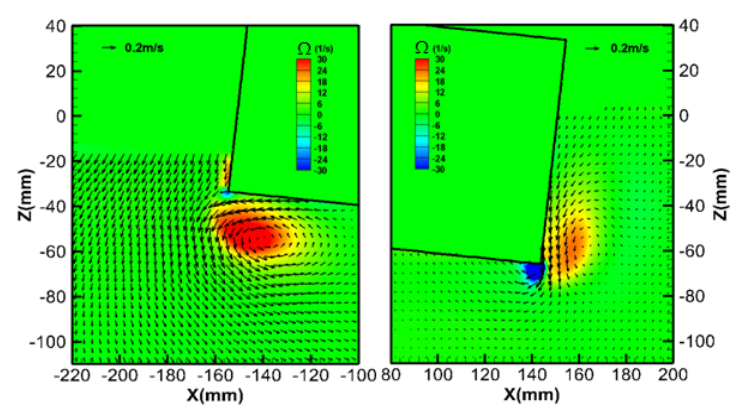

(a)

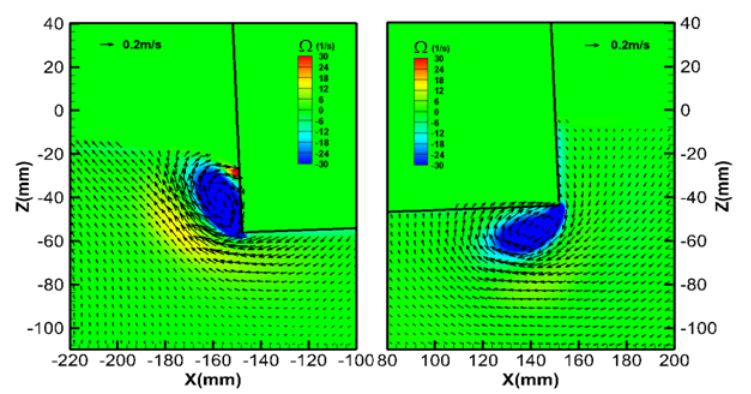

(c)

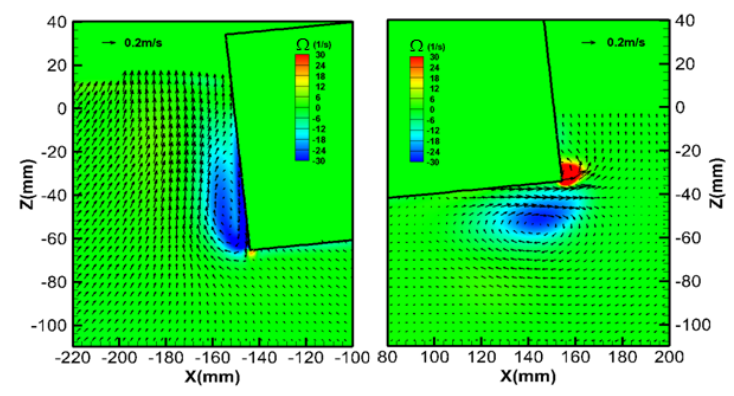

(e)

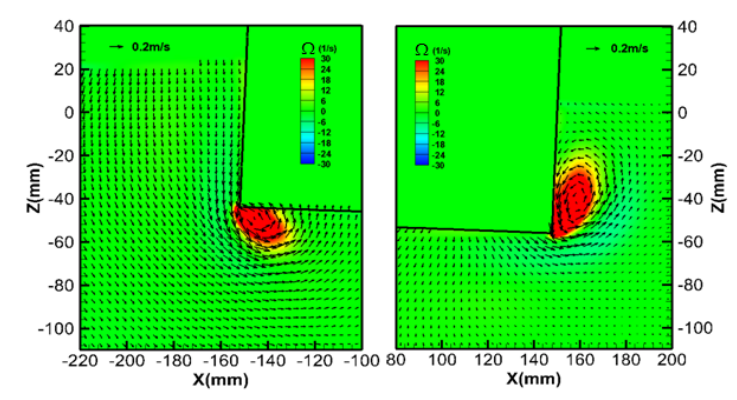

(g)

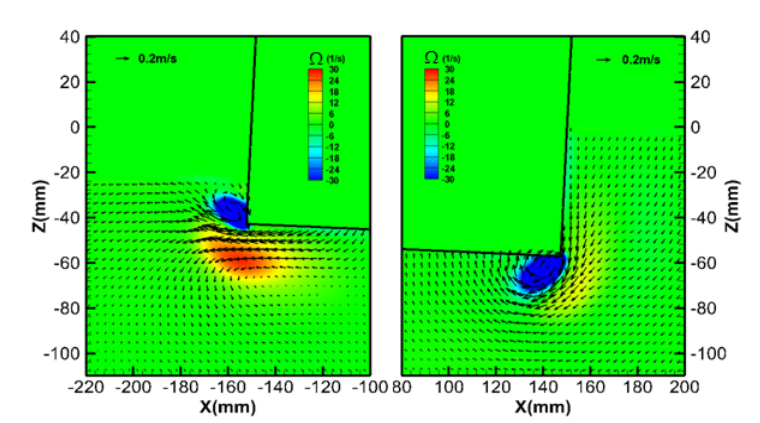

(b)

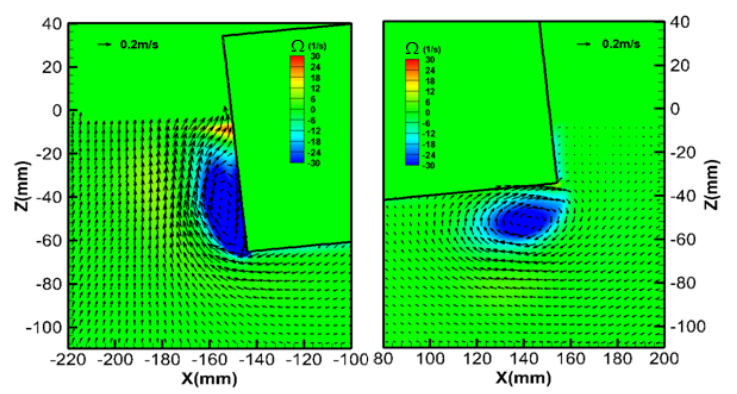

(d)

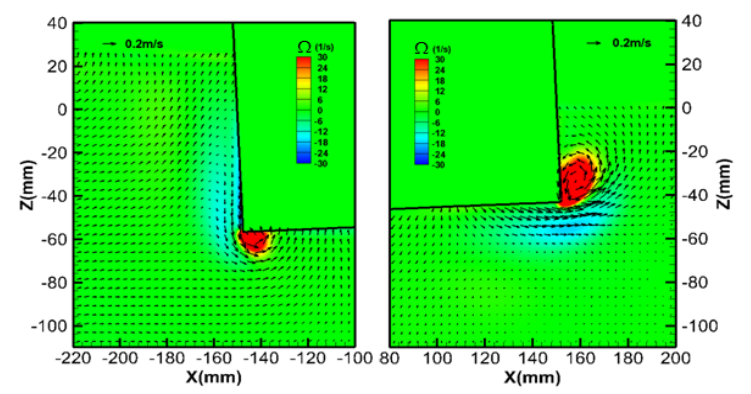

(f)

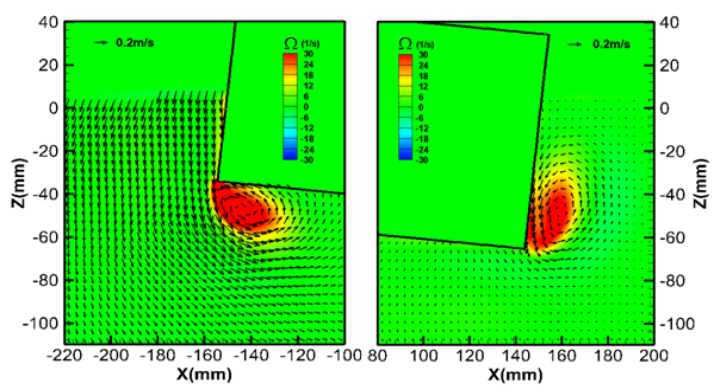

(h)

Fig. 7 Velocity field of $T_{s}=0.8 \mathrm{~s}$ wave : phase number of each subtitle matches to the phase in Fig. 3(b). 


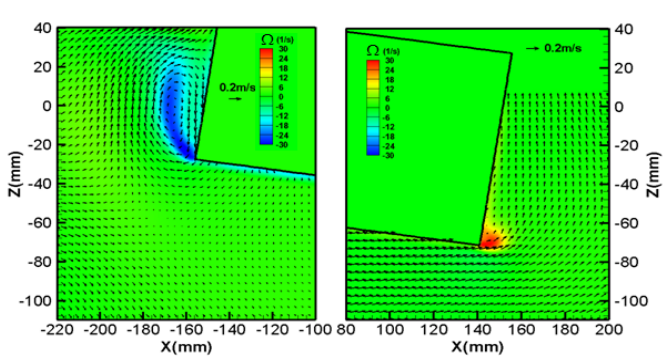

(a)

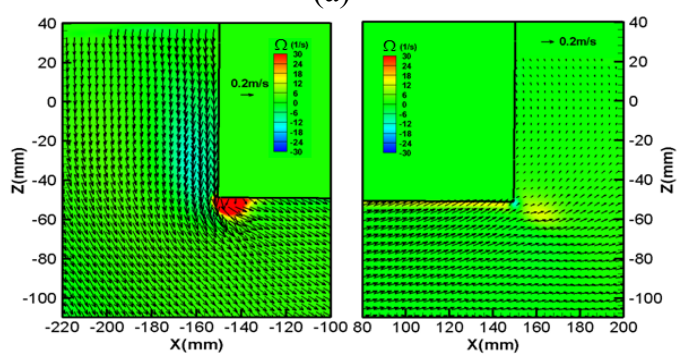

(c)

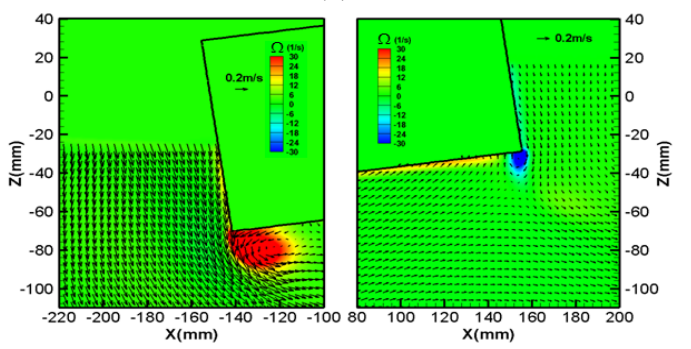

(e)

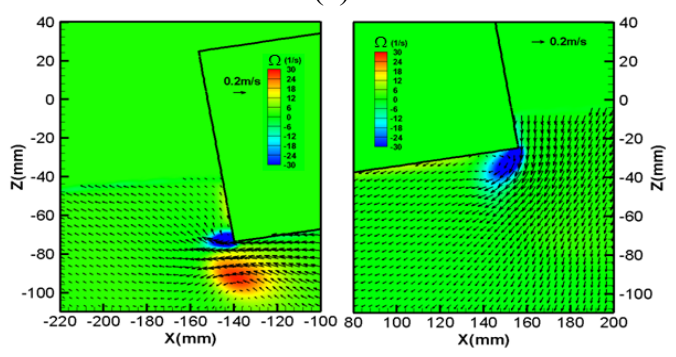

(g)

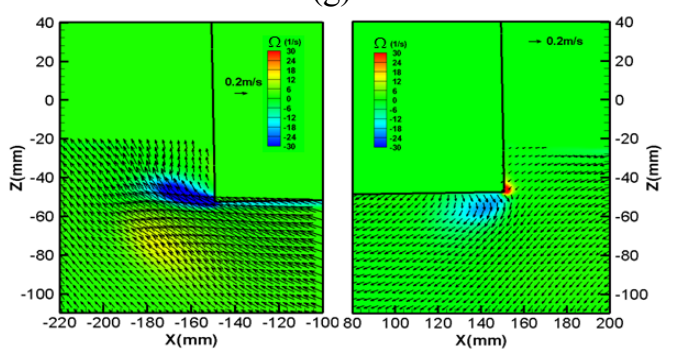

(i)

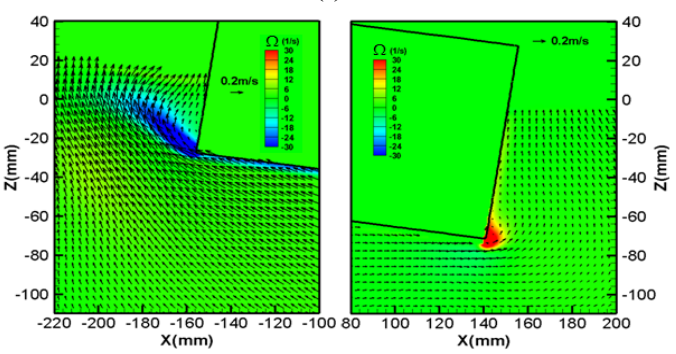

(k)

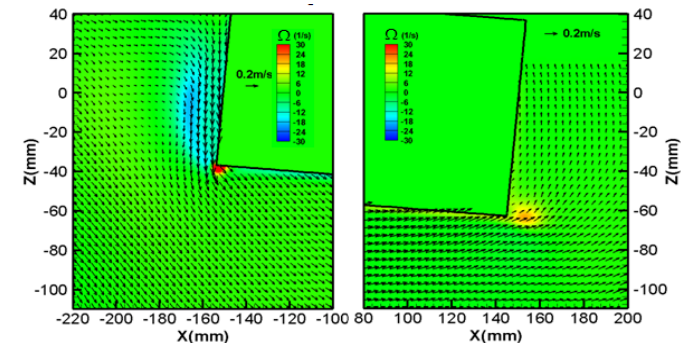

(b)

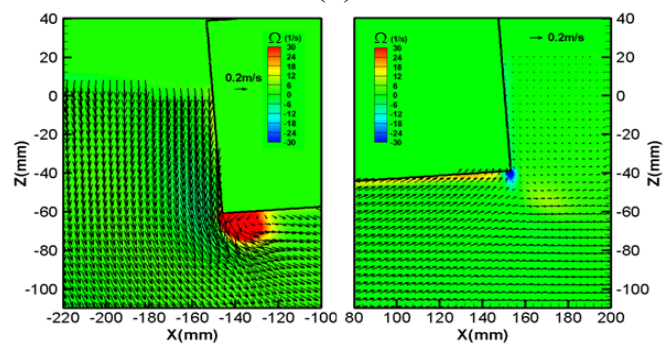

(d)

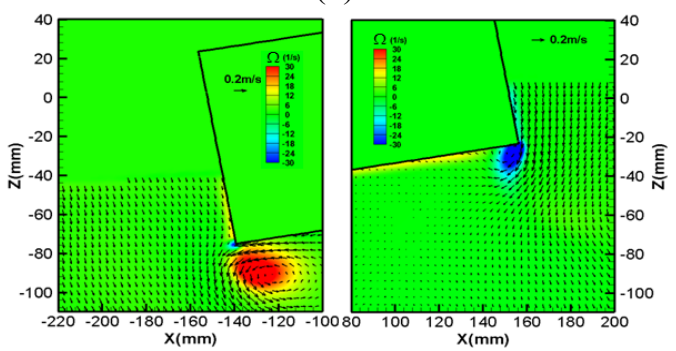

(f)

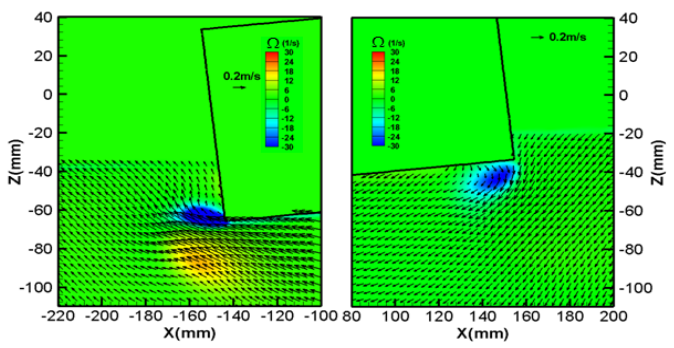

(h)

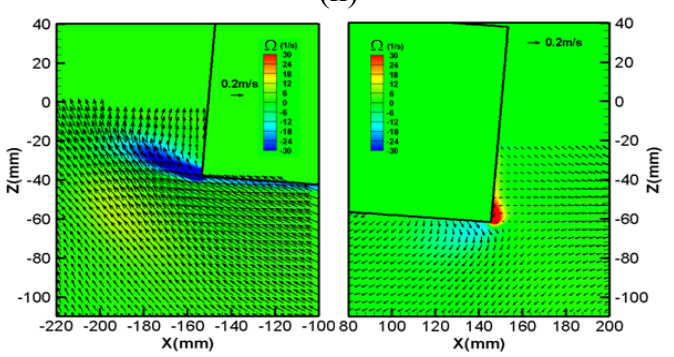

(j)

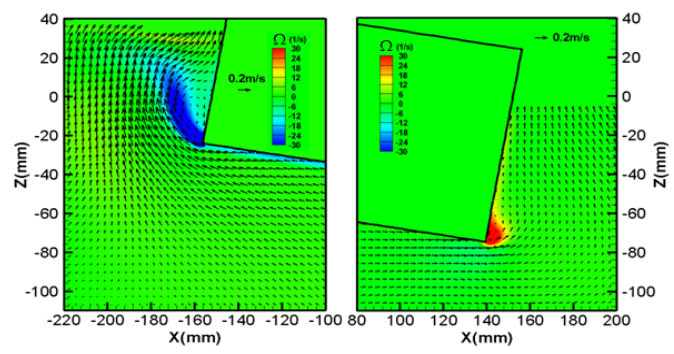

(1)

Fig. 8 Velocity field of $T_{s}=1.2 \mathrm{~s}$ wave : phase number of each subtitle matches to the phase in Fig. 3(c). 
negative vorticity developed in a circular shape at the trough of a wave on the seaward side in phase 2 , the negative vortex began to be separated by counterclockwise roll motion on the leeward side, as depicted in Fig. 7(b).

As the roll motion increased in the counterclockwise direction, the negative vortex with negative vorticity increased in size and strength on the seaward side. Also, the negative vortex near the right side bottom on the leeward side developed in phase 3 and phase 4, as shown in Figs. 7(c) and (d), respectively.

After passing the extreme positive rotational angle, the clockwise rotating vortex near the right bottom corner on the leeward side of the structure moved away from the structure with decreasing size and strength in phase 5, as shown in Fig. 7(e). However, a tiny positive vorticity appeared in the right corner on the leeward side. The clockwise rotating vortex on the seaward side decayed in this phase due to the clockwise roll motion.

Beyond phase 6 , the clockwise roll motion accompanying the descending free surface contributed to the generation of a positive vortex on the seaward side as shown in Fig. 7(f). Also, the positive vortex was evolved by the roll motion as clarified in phases 7 and 8 in Figs. 7(g) and (h), respectively. On the leeward side, the positive vortex increased with increased magnitude of the positive vorticity in the corner with increased the roll motion in the clockwise direction, corresponding to phases 6,7 and 8 in Figs. 7(f), (g) and (h), respectively.

At this wave period of $T_{s}=0.8 \mathrm{~s}$, the corner vorticies perform the same viscous damping effect on the roll motion with the case of $T_{s}=0.93 \mathrm{~s}$. Also, the presence of the corer vortices contribute to forming the negative pressure, like the case of $T_{s}=$ $0.93 s$ shown in Fig. 6.

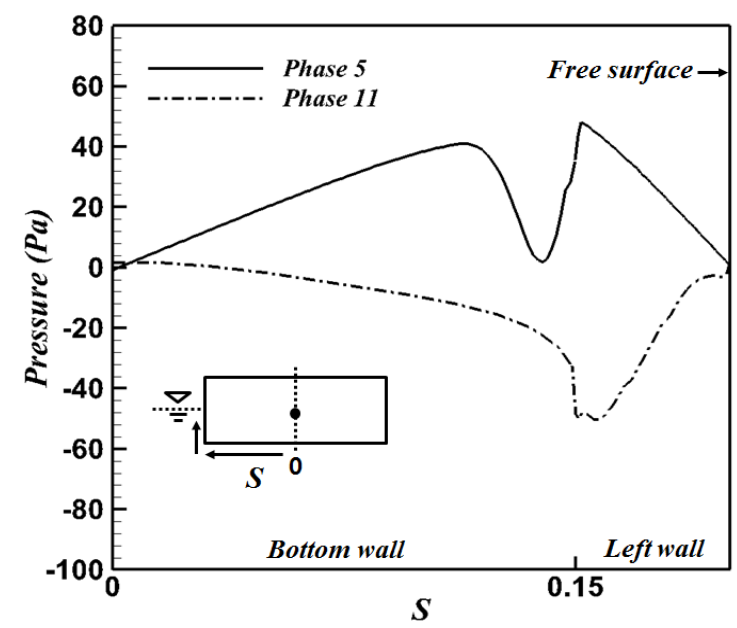

Fig. 9 Pressure distributions along the structure surface in phases 5 and 11 at $T_{s}=1.2 \mathrm{~s}$.

Fig. 8 shows the velocity vectors and the vorticity contours at different phases for a wave period of $T_{s}=1.2 \mathrm{~s}$, which is longer than the roll natural period. The longer wave period of $T_{s}=1.2 \mathrm{~s}$ reveals a different mechanism of the vortex evolution near each bottom corner of the structure compared to the shorter wave period cases of $T_{s}=0.93 \mathrm{~s}$ and $T_{s}=0.8 \mathrm{~s}$. At the shorter wave periods of $T_{s}=0.93 \mathrm{~s}$ and $T=0.8 \mathrm{~s}$, the development of the vortex near both bottom corners mainly depended on the roll motion of the structure as observed in Figs. 5 and 7, respectively.

However, with a longer wave of $T_{s}=1.2 \mathrm{~s}$ (compared to the roll natural period), the seaward and leeward side bottom corners are different from each other in terms of the vortex evolution mechanism. Near the left bottom corner on the seaward side, the vortex was mainly generated by water motion. In contrast, near the right bottom corner on the seaward side, the development of the vortex depended on the roll motion of the structure as in the cases of the shorter wave period of $T_{s}=0.93 \mathrm{~s}$ and $T_{s}=0.8 s$.

When the structure rotated in the counterclockwise direction, corresponding to phases (1-6) as shown in Figs. 8(a)-(f), respectively, the free surface on the seaward side had the highest level in phase 2 and the incident wave was the crest in phase 3 , which is shown on Fig. 3(c). On the seaward side the free surface descending to the minimum water level was faster than the rotation of the structure corner in the counterclockwise direction. Then, the positive vortex most developed with decaying the negative vortex under the structure on the seaward side with descending the water level. This vortex pattern is the opposite of those of the shorter wave periods of $T_{s}=0.93 \mathrm{~s}$ and $T_{s}=0.8 \mathrm{~s}$. 
At the corner of the leeward side, with the structure rotating in the counterclockwise direction, the remnant positive vortex decayed in phases (1-3) as shown in Figs. 8(a)-(c), respectively. However the negative vortex initiated in phase 4 (Fig. 8(d)) and increased in the phases (5-6) in Figs. 8(e)-(f), respectively. Thus, this comes to conclusion that the roll motion was predominant to the vortex evolution in the corner of the leeward side for the longer wave $T_{s}=1.2 \mathrm{~s}$.

When the structure rotated in the clockwise direction with the free surface ascending in phases (7-12) in Figs. 8(g)-(1), respectively, the negative vortex developed while the positive vortex dissipated away from the bottom corner on the seaward side. In contrast to the seaward side, the positive vortex developed while the negative vortex dissipated near the left bottom corner on the leeward side.

Fig. 9 shows the pressure distribution on the body surface in representatively phases 5 and 11 where the corresponding velocity field are in Figs. 8(e) and (k), respectively. Like Fig. 6, the hydrostatic pressure is ignored to observe the net effect of the vortices and the left half of the body is only considered. In phases 5 and 11, the low pressures appear near the left bottom corner and the position near the left corner on the left side wall, respectively, which are associated with the strong counterclockwise and clockwise rotating vortices at these regions as shown in Figs. Figs. 8(e) and (k). In these phases, these low pressures play a role in boosting the counterclockwise and clockwise roll motion of the body. This contribution of the corner vortices on the roll motion of the body at this $T_{s}=1.2 \mathrm{~s}$ is opposite to the case of $T_{s}=0.93 \mathrm{~s}$ as early explained in Fig. 6. Consequently, the present numerical results are in very good agreement with the experimental results of Jung (2004b) for all wave periods considered in this study.

\section{Forces}

Fig. 10 shows the x-directional forces $\left(F_{\mathrm{x}}\right)$ with wave elevations of $\eta_{S}$ and $\eta_{L}$ on the seaward and leeward sides, respectively, according to different wave periods. The sign and magnitude of $F_{\mathrm{x}}$ were mainly determined by the difference in wave heights on the seaward and on leeward sides. This means that the surface normal to the $\mathrm{x}$-direction, which was exposed to the hydrostatic pressure, determined the sign and magnitude of $F_{\mathrm{x}}$. Thus, when $\eta_{S}$ minus $\eta_{L}$ has a positive value, $F_{\mathrm{x}}$ is positive. The opposite is also true. This relation between $F_{\mathrm{x}}$ and the difference in the wave elevations is clarified from Figs. 10(a-c) for $T_{s}=0.93 s, T_{s}=0.8 s$ and $T_{s}=1.2 s$, respectively.

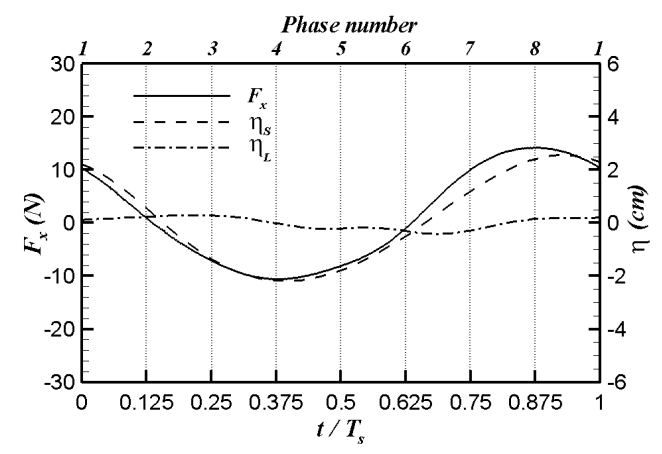

(a)

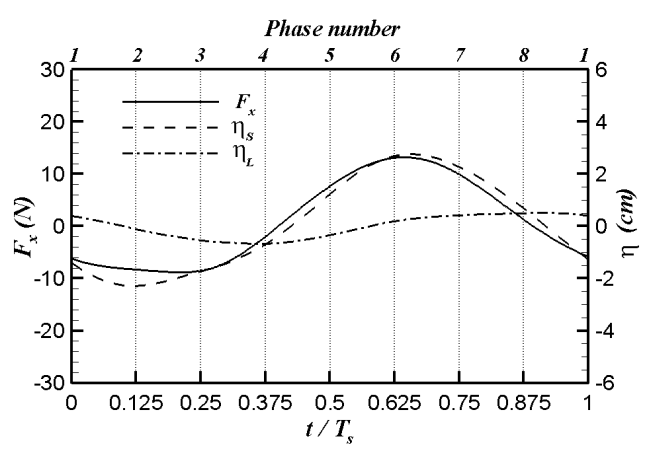

(b)

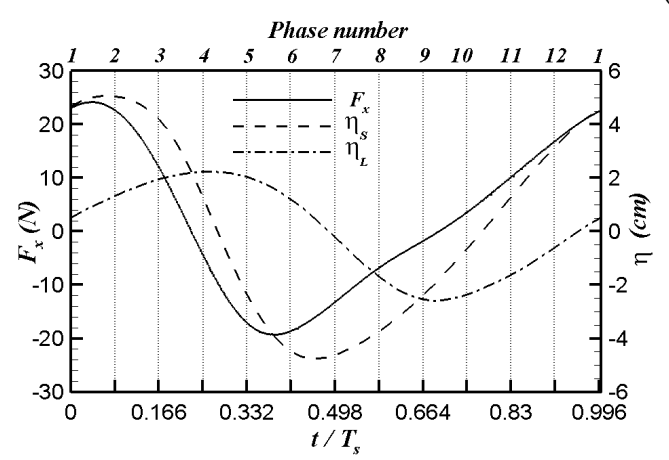

(c)

Fig. 10 Time histories of drag force with phases of velocity field at (a) $T_{s}=0.93 \mathrm{~s}$, (b) $T_{s}=0.8 \mathrm{~s}$ and (c) $T_{s}=1.2 \mathrm{~s}$ (solid line : drag force; dashed line : wave at the front of structure; dash-dotted line : wave behind structure). 
Fig. 11 shows the z-directional forces $\left(F_{z}\right)$ with the wave elevations of $\eta_{S}$ and $\eta_{L}$ on the seaward and leeward sides, respectively, according to different wave periods in one periods. As expected, the time behavior of $F_{z}$ was determined by the descent and the ascent of the wave. Especially, in the cases of $T_{S}=0.93 \mathrm{~s}$ and $T_{S}=0.8 \mathrm{~s}$, since the variation of $\eta_{L}$ on the leeward side was not significant, the change in $\eta_{S}$ led to the time behavior of $F_{z}$ as shown in Figs. 11(a) and (b) for $T_{s}=$ $0.93 \mathrm{~s}$ and $T_{s}=0.8 \mathrm{~s}$, respectively.

The maximum and minimum values of $F_{x}$ and $F_{z}$ are plotted in Figs. 12(a) and (b), respectively. The variations in the maximum and the minimum values of $F_{x}$ and $F_{z}$ between the natural wave period of $T_{s}=0.93 \mathrm{~s}$ and the shorter wave period of $T_{s}=0.8 \mathrm{~s}$ are minor. However, as the wave period increased from the natural wave period of $T_{s}=0.93 \mathrm{~s}$ to $T_{s}=1.2 \mathrm{~s}$, the maximum and the minimum values of both forces rapidly increased.

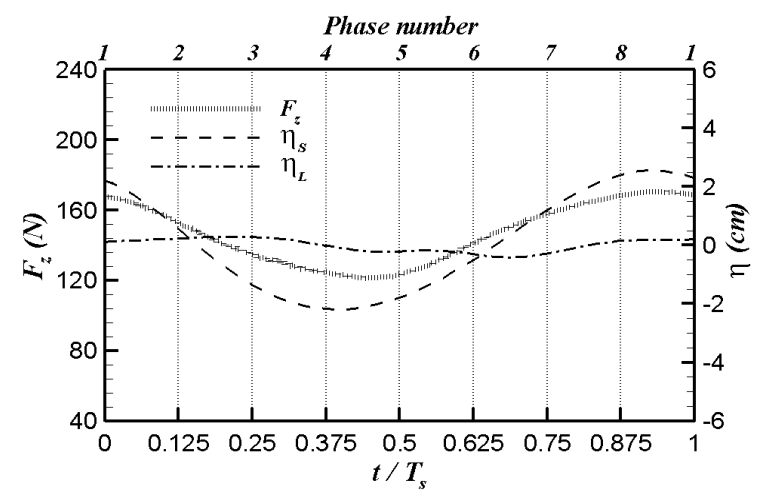

(a)

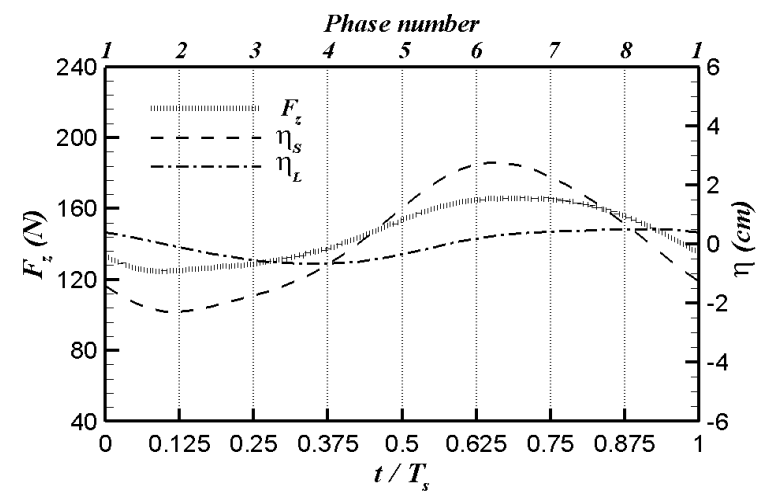

(b)

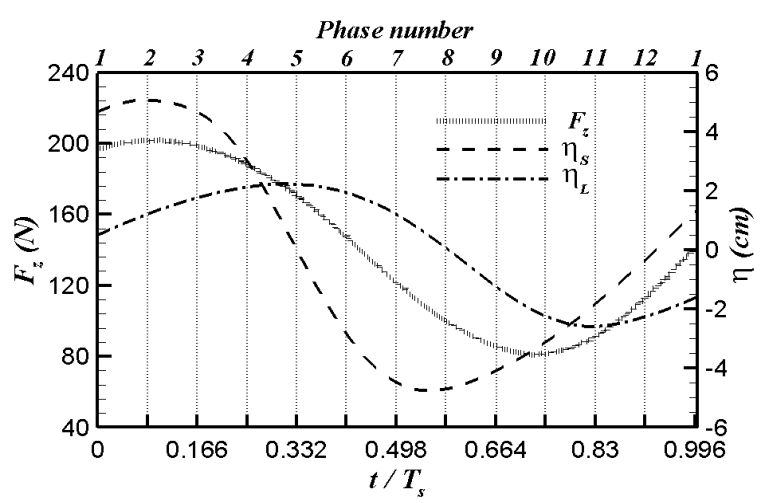

(c)

Fig. 11 Time histories of lift force with phases of velocity field at (a) $T_{s}=0.93 \mathrm{~s}$, (b) $T_{s}=0.8 \mathrm{~s}$ and (c) $T_{s}=1.2 \mathrm{~s}$ (dotted line : lift force; dashed line : wave at the front of structure; dash-dotted line : wave behind structure).

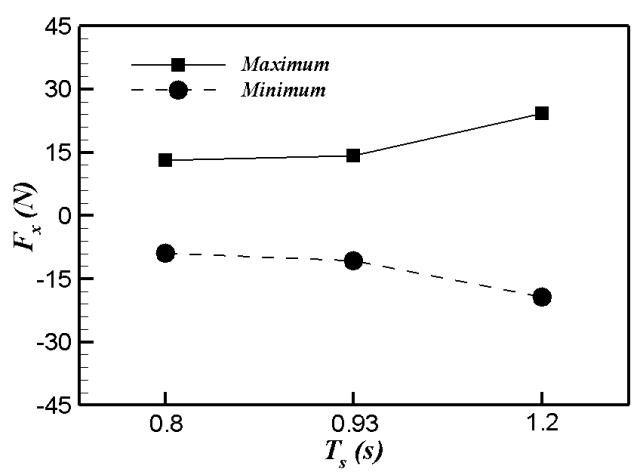

(a)

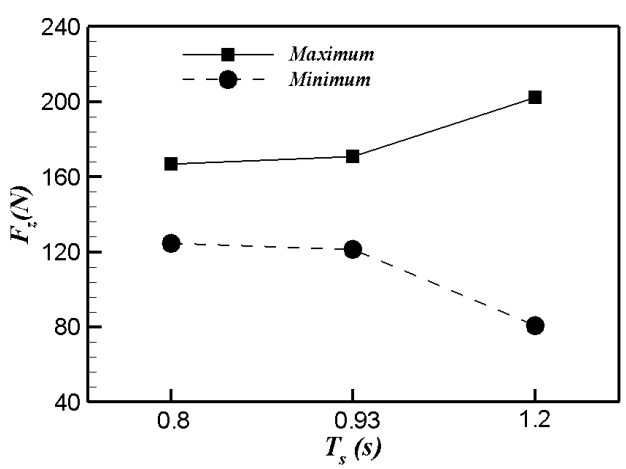

(b)

Fig. 12 The maximum and minimum of the $\mathrm{x}$ and $\mathrm{z}$-directional force as a function of the wave period $\left(T_{s}\right)$. 


\section{CONCLUSIONS}

This study numerically investigated the interaction of a regular wave and the roll motion of a rectangular floating structure. In order to simulate the two-dimensional (2D) incompressible viscous two-phase flow in a numerical wave tank with the rectangular floating structure, the present study used the volume of fluid (VOF) method based on the finite volume method. The sliding mesh technique was adopted to characterize the motion of the rectangular floating structure induced by fluid-structure interaction. The effects of the wave period on the flow, roll motion and forces acting on the structure were examined by considering three different wave periods that were shorter, equal to, and longer than roll natural period of the rectangular structure.

The time variations of the wave height and the roll motion of the rectangular structure are in good agreement with those of Jung's experimental results (2004b) for all wave periods. The present RAOs compare well with the experimental results. Also, the present RAOs compare well with linear potential theory except at the natural frequency where the RAO based on theory is significantly magnified.

The present numerical results represented well the entire process of vortex generation and evolution presented by Jung (2004b). The shorter and natural wave periods revealed that the viscous damping effect reduced the roll motion. At a longer wave period, development of the vortex depended on the wave level instead of the roll motion shown in the shorter wave periods.

The x-directional forces were mainly governed by the difference in wave heights on the seaward and leeward sides. The time behavior of the z-directional forces was determined by the descent and the ascent of the wave. As the wave period increased beyond the natural wave period, the maximum and the minimum values of both forces rapidly increased.

\section{ACKNOWLEDGEMENT}

This work was supported by the National Research Foundation of Korea (NRF) grant funded by the Korea government (MSIP) through GCRC-SOP (No. 2011-0030013) and (No. 2010-0025618).

\section{REFERENCES}

Chen, H.C., Liu, T., Chang, K.A. and Huang, E.T., 2002. Time-domain simulation of barge capsizing by a Chimera Domain Decomposition Approach. Proceedings of the 12th International Offshore and Polar Engineering Conference. Kitakyushu, Japan, 26-31 May 2002, pp.26-31.

Downie, M.J., Bearman, P.W. and Graham, J.M.R., 1988. Effect of vortex shedding on the coupled roll response of bodies in waves. Journal of fluid mechanics, 189, pp.243-261.

Faltinsen, O.M. and Pettersen, B., 1987. Application of a vortex tracking method to separated flow around marine structures. Journal of Fluids and Structures, 1(2), pp.217-237.

FLUENT, 2010. ANSYS FLUENT user's guide. Canonsburg, USA: ANSYS Inc.

Himeno, Y., 1981. Prediction of ship roll damping-state of the art, Rep no. 239: Department of Naval Architecture, University of Michigan, Michigan.

Hirt, C.W. and Nichols, B.D., 1981. Volume of fluid (VOF) method for the dynamics of free boundaries. Journal of Computational Physics, 39, pp.201-225.

Ikeda, Y. and Himeno, Y., 1981. Calculation of vortex-shedding flow around oscillating circular and Lewis-form cylinder. Proceedings of the 3rd International Conference on Numerical Ship Hydrodynamics. Paris, France, 16-19 June 1981.

Jung, K.H., Chang, K.A. and Huang, E.T., 2004a. Two-dimensional flow characteristics of wave interactions with a fixed rectangular structure. Ocean Engineering, 31, pp.975-998.

Jung, K.H., 2004b. Experimental study on rectangular barge in beam sea. Ph.D. Texas A\&M University.

Jung, K.H., Chang, K.A. and Huang, E.T., 2005. Two-dimensional flow characteristics of wave interactions with a free-rolling rectangular structure. Ocean Engineering, 32(1), pp.1-20.

Korpus, R.A. and Falzarano, J.M., 1997. Prediction of viscous ship roll damping by unsteady Navier-Stokes techniques. Journal of Offshore Mechanics and Arctic Engineering, Transactions of the ASME, 119(2), pp.108-113.

Launder, B.E. and Spalding, D.B., 1972. Lectures in mathematical models of turbulence. London, England: Academic Press. 
Salvesen, N., Tuck, E.O. and Faltinsen, O.M., 1970. Ship motions and sea loads. Transactions of SNAME, 78, pp.250-287.

Sarkar, T. and Vassalos, D.A., 2000. A RANS-based technique for simulation of the flow near a rolling cylinder at the free surface. Journal of Marine Science and Technology, 5, pp.66-77.

Scolan, Y.M. and Faltinsen, O.M., 1994. Numerical studies of separated flow from bodies with sharp corners by the vortex in cell method. Journal of Fluids and Structures, 8(2), pp.201-230.

Wehausen, J.V., 1971. The motion of floating bodies. The Annual Review of Fluid Mechanics, 3, pp.237-268.

Wilson, R.V., Carrica, P.M. and Stern, F., 2006. Unsteady RANS method for ship motions with application to roll for a surface combatant. Computers and Fluids, 35(5), pp.501-524.

Yeung, R.W. and Vaidhyanathan, M., 1994. Highly separated flows near a free surface. Proceedings of the International Conference on Hydrodynamics. Wuxi, China, 30 October - 3 November 1994. 